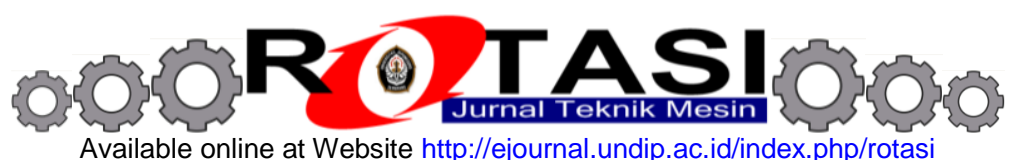

\title{
KONTROL SUDUT ATTITUDE MENGGUNAKAN LINEAR QUADRATIC REGULATOR (LQR) UNTUK QUADROTOR DENGAN PAYLOAD
}

\author{
* Mochammad Ariyanto, Joga Dharma Setiawan, Ismoyo Haryanto \\ Program Studi Magister Teknik Mesin, Fakultas Teknik, Universitas Diponegoro \\ Jl. Prof. Sudharto, SH., Tembalang-Semarang 50275, Telp. +62247460059 \\ *E-mail: ari_janto5@yahoo.co.id
}

\begin{abstract}
ABSTRAK
Quadrotor telah dikembangkan oleh peneliti di dunia ini sebagai aerial object interaction/aerial manipulation. Agar quadrotor bisa terbang mengangkat beban, maka dibutuhkan sistem kontrol yang dapat mengkompensasi perubahan pusat gravitasi. Pada penelitian ini, model matematika quadrotor dengan efek beban/payload akan dikembangkan. Untuk mengkompensasi perubahan lokasi pusat gravitasi karena efek beban, Linear Quadratic Regulator (LQR) akan digunakan untuk stabilisasi sudut Euler.Model linear state space dihitung menggunakan persmaan gerak onlinear quadrotor tanpa beban, kemudian disintesis sistem kontrol LQR. Kontrol tersebut diterapkan dalam model matematika nonlinear dengan beban/payload. Berdasarkan hasil simulasi integral action dari kontrol LQR dapat menghilangkan steady state error setelah diberikan step input, dan kondisi sudut awal sebesar $5^{0}$. Variasi beban dari 0 gr sampai dengan 200 gr tidak akan memberikan steady state error.

Kata kunci: attitude, beban, LQR, quadrotor, state space
\end{abstract}

\section{PENDAHULUAN}

Pada saat ini penelitian tentang sistem kontrol quadrotor telah berkembang pesat. Di universitas Pennsylvania [1], telah megembangkan quadrotor yang dilengkapi gripper untuk mengangkat object yang ringan. Di universitas Utah, telah mengembangkan quadrotor untuk aerial manipulation dengan menggunakan kontrol PID[2]. Palunko, [3] telah mengembangkan model matematika quadrotor dengan perubahan lokasi pusat gravitasi dan membuat control adaptive untuk mengkompensasi posisi pusat gravitasi tersebut. Agar quadrotor bisa terbang mengangkat beban, maka dibutuhkan sistem kontrol yang dapat mengkompensasi perubahan pusat gravitasi. Pada pemodelan quadrotor tanpa beban dapat dijumpai pada literatur [4], dan [5]. Pada referensi [6], dan [7] telah mengembangkan model matematika pada underwater vehicle disertai dengan lokasi pusat gravitasi. Pada penelitian ini, model matematika quadrotor dengan efek beban/payload akan dikembangkan. Untuk mengkompensasi perubahan lokasi pusat gravitasi karena beban, Linear Quadratic Regulator (LQR) akan digunakan untuk stabilisasi sudut Euler. Integral action pada LQR akan digunakan untuk menghilangkan steady state error pada sudut Euler. Kontrol LQR untuk stabilisasi sudut Euler dapat dijumpai pada referensi [8], dan [9].

\section{MODEL MATEMATIKA QUADROTOR DENGAN PAYLOAD}

Bagian ini akan membahas pemodelan dinamika quadrotor dilengkapi dengan gripper pada saat membawa beban/payload dalam kondisi terbang hover. Dalam pemodelan dinamika nonlinier quadrotor dengan beban, menggunakan asumsi / penyederhanaan sebagai berikut:

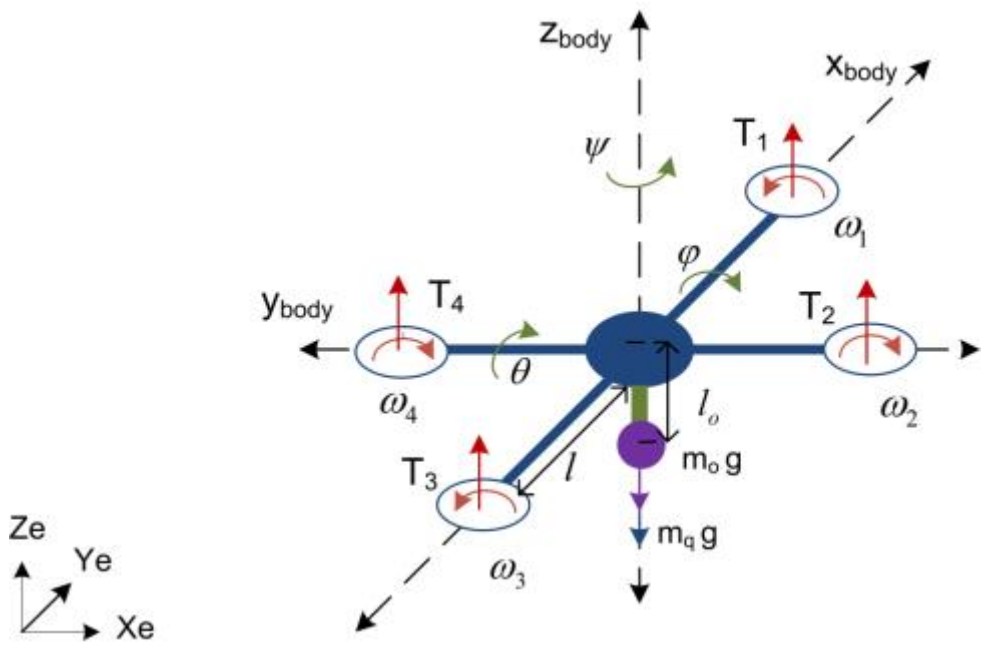

Gambar 1. Sistem koordinat quadrotor saat membawa beban 
- $\quad$ Struktur Quadrotor rigid dan simetris

- Quadrotor dan beban dimodelkan sebagai lump mass yang mempengaruhi posisi pusat massa atau pusat gravitasi tidak bertepatan dengan pusat quadrotor pada koordinat badan tetap quadrotor

- $\quad$ Struktur empat baling-baling yang kaku

- Gaya angkat linier dengan tegangan input, empat brushless DC motor memiliki respon bandwidth tinggi sehingga pemodelan dinamis aktuator tidak diperlukan dan dimodelkan sebagai persamaan aljabar

- Gaya drag yang melawan quadrotor diabaikan karena quadrotor terbang pada kondisi hover

Gerakan sebuah Quadrotor yang memiliki 6 derajat kebebasan, dua frame koordinat didefinisikan sebagai yang ditunjukkan dalam Gambar 1 dan Gambar 2. Kerangka koordinat bergerak $\{\mathrm{B}\}$ adalah tetap pada badan quadrotor dan ini disebut sebagai koordinat badan quadrotor. Asal dari kerangka quadrotor dipilih bertepatan dengan Center of Gravity (COG) ketika CoG berada dalam principal plane quadrotor. Tapi, ketika Quadrotor membawa beban, maka akan menyebabkan pusat gravitasi atau pusat massa tidak bertepatan dengan simetri dari principal plane quadrotor, seperti yang ditunjukkan pada Gambar 2. Gerakan Quadrotor digambarkan relatif terhadap koordinat tetap di bumi (inertial fixed frame) $\{\mathrm{G}\}$.

Untuk quadrotor, diasumsikan bahwa percepatan suatu titik pada permukaan bumi dapat diabaikan. Sebagai hasil dari ini, koordinat tetap di bumi $\{\mathrm{G}\}$ dianggap inersia. Posisi dan orientasi quadrotor relatif terhadap koordinat tetap di bumi $\{G\}$. Kinematika quadrotor dideskripsikan dalam variabel-variabel ini, persamaan (1) merupakan posisi koordinat badan quadrotor $\{B\}$ diukur dalam koordinat tetap di bumi $\{G\}$, persamaan (2) mengungkapkan sudut Euler dari $\{B\}$ terhadap $\{G\}$, di mana persamaan (3) dan (4) adalah kecepatan translasi dan kecepatan sudut pada koordinat badan quadrotor.

$$
\begin{aligned}
& \eta_{1}=\left[\begin{array}{lll}
x & y & z
\end{array}\right]^{T} \\
& \eta_{2}=\left[\begin{array}{lll}
\varphi & \theta & \psi
\end{array}\right]^{T} \\
& v_{1}=\left[\begin{array}{lll}
u & v & w
\end{array}\right]^{T} \\
& v_{2}=\left[\begin{array}{lll}
p & q & r
\end{array}\right]^{T}
\end{aligned}
$$

Hubungan antara vektor kecepatan pada koordinat tetap di bumi $\left(\dot{\eta}_{1}\right)$ dan vector kecepatan pada koordinat bodan quadrotor $v_{1}$ dapat dihitung menggunakan persamaan (5).

$$
\dot{\eta}_{1}=\frac{d \eta_{1}}{d t}={ }_{B}^{G} R\left(\eta_{2}\right) v_{1}
$$

Di mana matriks ${ }_{B}^{G} R\left(\eta_{2}\right)$ merupakan matriks transformasi dari koordinat badan ke koordinat tetap di bumi yang diperoleh dari perkalian matriks tiga rotasi orthogonal dasar.

$$
\left[\begin{array}{c}
\dot{x} \\
\dot{y} \\
\dot{z}
\end{array}\right]=\left[\begin{array}{ccc}
c \psi c \theta & -s \psi c \phi+c \psi s \theta s \phi & s \psi s \phi+c \psi s \theta c \phi \\
s \psi c \theta & c \psi c \phi+s \psi s \theta s \theta & -c \psi s \phi+s \psi s \theta c \phi \\
-s \theta & c \theta s \phi & c \theta c \phi
\end{array}\right]\left[\begin{array}{c}
u \\
v \\
w
\end{array}\right]
$$
dan (9).

Transformasi dari angular body rates ke dalam kecepatan sudut Euler dapat ditulis dalam persamaan (7), (8),

$$
\begin{aligned}
& \dot{\eta}_{2}=\frac{d \eta_{2}}{d t}=Q\left(\eta_{2}\right) v_{2} \\
& Q\left(\eta_{2}\right)=\left[\begin{array}{ccc}
1 & s \varphi t \theta & c \varphi t \theta \\
0 & c \varphi & -s \varphi \\
0 & \frac{s \varphi}{c \theta} & \frac{c \varphi}{c \theta}
\end{array}\right]
\end{aligned}
$$


$\left[\begin{array}{c}\dot{\varphi} \\ \dot{\theta} \\ \dot{\psi}\end{array}\right]=\left[\begin{array}{ccc}1 & s \varphi t \theta & c \varphi t \theta \\ 0 & c \varphi & -s \varphi \\ 0 & \frac{s \varphi}{c \theta} & \frac{c \varphi}{c \theta}\end{array}\right]\left[\begin{array}{c}p \\ q \\ r\end{array}\right]$

Akhirnya, hubungan kinematika mentransformasikan dari koordinat badan tetap quadrotor $\{\mathrm{B}\}$ ke dalam koordinat tetap di bumi $\{\mathrm{G}\}$ dapat dihitung dengan menggunakan persamaan (10)

$\left[\begin{array}{l}\dot{\eta}_{1} \\ \dot{\eta}_{2}\end{array}\right]=\left[\begin{array}{cc}{ }_{B}^{G} R\left(\eta_{2}\right) & 0 \\ 0 & Q\left(\eta_{2}\right)\end{array}\right]\left[\begin{array}{l}v_{1} \\ v_{2}\end{array}\right]$

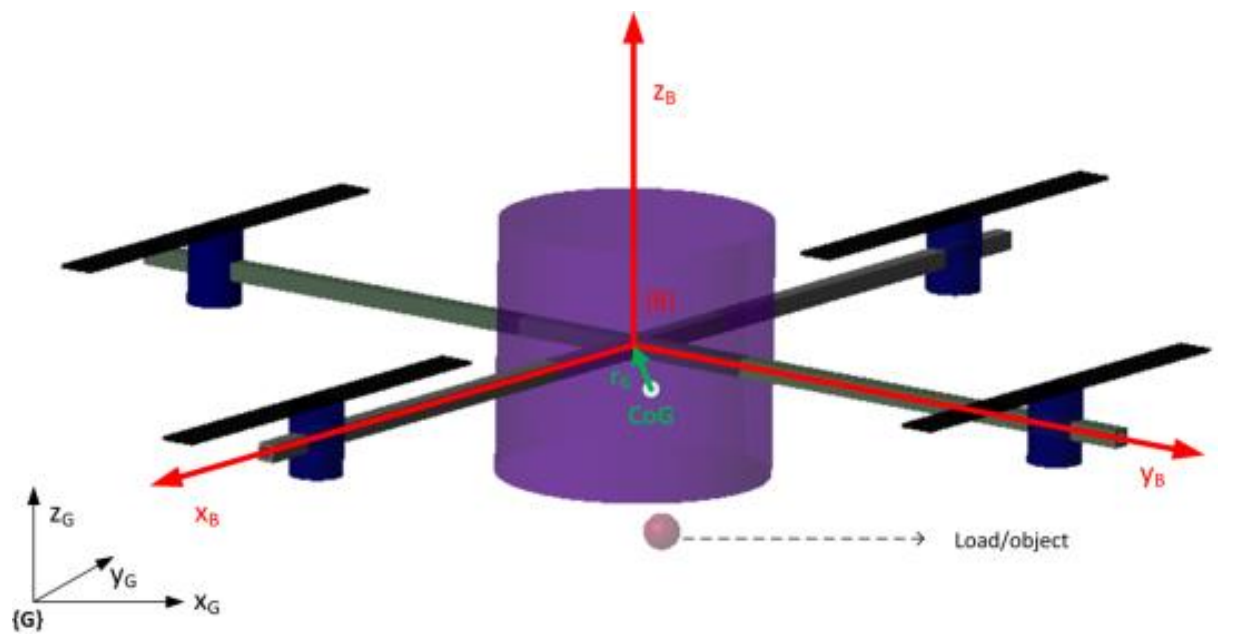

Gambar 2. Perubahan posisi pusat gravitasi quadrotor karena payload

Posisi CoG yang baru terhadap koordinat badan quadrotor $\{\mathrm{B}\}$ dapat dituliskan sebagai berikut

$$
\begin{aligned}
& l_{o}=\left[\begin{array}{lll}
l_{o x} & l_{o y} & l_{o z}
\end{array}\right]^{T} \\
& r_{G}=\left[\begin{array}{lll}
x_{G} & y_{G} & z_{G}
\end{array}\right]^{T} \\
& x_{G}=\frac{m_{o} l_{o x}}{m_{q}+m_{o}} \\
& y_{G}=\frac{m_{o} l_{o y}}{m_{q}+m_{o}} \\
& z_{G}=\frac{m_{o} l_{o z}}{m_{q}+m_{o}}
\end{aligned}
$$

Dengan vector $\mathrm{r}_{\mathrm{G}}$ merepresentasikan posisi pusat gravitasi yang diukur dari koordinat badan quadrotor vector percepatan pada CoG pada koordinat badan quadrotor adalah sebagai berikut ini

$$
\begin{aligned}
& F x=m \dot{u}-m v r+m w q-m x_{G}\left(q^{2}+r^{2}\right)+m y_{G}(p q-\dot{r})+m z_{G}(p r+\dot{q}) \\
& F y=m \dot{v}-m w p+m u r+m x_{G}(q p+\dot{r})-m y_{G}\left(p^{2}+r^{2}\right)+m z_{G}(q r-\dot{p}) \\
& F z=m \dot{w}-m u q+m v p+m x_{G}(r p-\dot{q})+m y_{G}(r q-\dot{p})-m z_{G}\left(q^{2}+p^{2}\right) \\
& \tau_{x}=I_{x x} \dot{p}+\left(I_{z z}-I_{y y}\right) q r+m\left[y_{G}(\dot{w}-u q+v p)-z_{G}(\dot{v}-w p+u r)\right] \\
& \tau_{y}=I_{y y} \dot{q}+\left(I_{x x}-I_{z z}\right) r p+m\left[z_{G}(\dot{u}-v r+w q)-x_{G}(\dot{w}-u q+v p]\right. \\
& \tau_{z}=I_{z z} \dot{r}+\left(I_{y y}-I_{x x}\right) p q+m\left[x_{G}(\dot{v}-w p+u r)-y_{G}(\dot{u}-v r+w q]\right.
\end{aligned}
$$


Matriks representasi persamaan gerak dinamik nonlinear quadrotor dengan beban/payload dapat dinyatakan dalam bentuk yang lebih kompak seperti yang tertulis dalam persamaan (22).

$M_{B} \dot{v}+C_{B}(v) v=G_{B}\left(\eta_{2}\right)+U_{B}$

Matriks inersia system adalah sebagai berikut

$$
M_{B} \dot{v}=\left[\begin{array}{cccccc}
m & 0 & 0 & 0 & m z_{G} & -m y_{G} \\
0 & m & 0 & -m z_{G} & 0 & m x_{G} \\
0 & 0 & m & -m y_{G} & -m x_{G} & 0 \\
0 & -m z_{G} & m y_{G} & I_{x x} & 0 & 0 \\
m z_{G} & 0 & -m x_{G} & 0 & I_{y y} & 0 \\
-m y_{G} & m x_{G} & 0 & 0 & 0 & I_{z z}
\end{array}\right]\left[\begin{array}{c}
\dot{u} \\
\dot{v} \\
\dot{w} \\
\dot{p} \\
\dot{q} \\
\dot{r}
\end{array}\right]
$$

Persamaan (24) merupakan matriks coriolis-centripetal

$$
C_{B}(v) v=\left[\begin{array}{c}
-m v r+m w q-m x_{G}\left(q^{2}+r^{2}\right)+m y_{G}(p q-\dot{r})+m z_{G}(p r+\dot{q}) \\
-m w p+m u r+m x_{G}(q p+\dot{r})-m y_{G}\left(p^{2}+r^{2}\right)+m z_{G}(q r-\dot{p}) \\
-m u q+m v p+m x_{G}(r p-\dot{q})+m y_{G}(r q-\dot{p})-m z_{G}\left(q^{2}+p^{2}\right) \\
\left(I_{z z}-I_{y y}\right) q r+m\left[y_{G}(\dot{w}-u q+v p)-z_{G}(\dot{v}-w p+u r)\right] \\
\left(I_{x x}-I_{z z}\right) r p+m\left[z_{G}(\dot{u}-v r+w q)-x_{G}(\dot{w}-u q+v p]\right. \\
\left(I_{y y}-I_{x x}\right) p q+m\left[x_{G}(\dot{v}-w p+u r)-y_{G}(\dot{u}-v r+w q]\right.
\end{array}\right]
$$

Dalam gerak translasi dan rotasi, gaya angkat yang dihasilkan oleh empat baling-baling pada koordinat badan quadrotor dan moment yang disebabkan oleh empat baling-baling pada $\mathrm{x}, \mathrm{y}, \mathrm{z}$ dalam koordinat badan quadrotor ditunjukkan dalam persamaan dari (25) ke (29). Input vektor dapat ditulis dalam persamaan (25)

$$
\begin{aligned}
& U_{B}=\left[\begin{array}{c}
0 \\
0 \\
U_{1} \\
U_{2} \\
U_{3} \\
U_{4}
\end{array}\right] \\
& \mathrm{U}_{\mathrm{B}}=\left[\begin{array}{ll}
F^{B} & \tau^{B}
\end{array}\right]^{T}=\left[\begin{array}{llllll}
F_{x} & F_{y} & F_{z} & \tau_{x} & \tau_{y} & \tau_{z}
\end{array}\right]^{T} \\
& U_{1}=F_{z}=F_{1}+F_{2}+F_{3}+F_{4} \\
& U_{2}=\tau_{x}=l\left(F_{4}-F_{2}\right) \\
& U_{3}=\tau_{y}=l\left(F_{3}-F_{1}\right) \\
& U_{4}=\tau_{z}=M_{2}+M_{4}-\left(M_{1}+M_{3}\right)
\end{aligned}
$$

Gaya dan momen yang dibangkitkan dari emat propeller adalah sebagai berikut

$$
\begin{aligned}
& F_{i}=a_{i} v_{i}+b_{i} \\
& M_{i}=c_{i} v_{i}+d_{i}
\end{aligned}
$$

Dimana $\mathrm{F}$ adalah gaya angkat motor dan baling-baling, sedangkan $\mathrm{M}$ adalah momena yang disebabkan oleh motor dan baling-baling, di mana $\mathrm{i}=1$ sampai 4 menunjukkan motor depan, kanan, belakang dan kiri. Koefisian a adalah koefisien dorong $(\mathrm{N} /$ volt), b gaya angkat konstan $(\mathrm{N})$, c adalah koefisien momen (Nm / volt), dan d adalah momen konstan $(\mathrm{Nm})$. 
Karena pusat gravitasi tidak bertepatan dengan koordinat tetap quadrotor,ini akan menyebabkan momen gravitasi ketika quadrotor dalam gerakan roll dan pitch. Transformasi gaya dan momen gravitasi dari koordinat tetap di bumi ke dalam koordinat badan quadrotor dapat dihitung menggunakan persamaan (30) dan (31)

$$
\begin{gathered}
f_{G}\left(\eta_{2}\right)={ }_{B}^{G} R^{-1}(\varphi, \theta)\left[\begin{array}{c}
0 \\
0 \\
-m g
\end{array}\right]=\left[\begin{array}{c}
m g \sin \theta \\
m g \cos \theta \sin \varphi \\
-m g \cos \theta \cos \varphi
\end{array}\right] \\
G_{B}\left(\eta_{2}\right)=\left[\begin{array}{c}
f_{G}\left(\eta_{2}\right) \\
r_{G} x f_{G}\left(\eta_{2}\right)
\end{array}\right]=\left[\begin{array}{c}
m g \sin \theta \\
m g \cos \theta \sin \varphi \\
{\left[\begin{array}{c}
x_{G} \\
y_{G} \\
z_{G}
\end{array}\right]\left[\begin{array}{c}
m g \cos \theta \cos \varphi \\
m g \sin \theta \\
m g \cos \theta \sin \varphi \\
-m \cos \theta \cos \varphi
\end{array}\right]}
\end{array}\right.
\end{gathered}
$$

Gaya dan momen gravitasi dapat dituliskan dalam persamaan (32)

$$
G_{B}\left(\eta_{2}\right)=\left[\begin{array}{c}
m g \sin \theta \\
m g \cos \theta \sin \varphi \\
-m g \cos \theta \cos \varphi \\
-m g y_{G} \cos \varphi \cos \theta+m g z_{G} \cos \varphi \cos \theta \\
m g z_{G} \sin \theta+m g x_{G} \cos \varphi \cos \theta \\
-m g y_{G} \sin \theta-m g x_{G} \cos \theta \sin \varphi
\end{array}\right]
$$

Representasi vektor persamaan gerak 6 derajat kebebasan dapat dilihat pada persamaan (33) dan (34)

$$
\begin{aligned}
& M_{B} \dot{v}+C_{B}(v) v=G_{B}\left(\eta_{2}\right)+U_{B} \\
& M_{B} \dot{v}=-C_{B}(v) v+G_{B}\left(\eta_{2}\right)+U_{B} \\
& M_{B} \dot{v}=M_{B}{ }^{-1}\left(-C_{B}(v) v+G_{B}\left(\eta_{2}\right)+U_{B}\right)
\end{aligned}
$$

$$
\dot{v}=\left[\begin{array}{cccccc}
m & 0 & 0 & 0 & m z_{G} & -m y_{G} \\
0 & m & 0 & -m z_{G} & 0 & m x_{G} \\
0 & 0 & m & -m y_{G} & -m x_{G} & 0 \\
0 & -m z_{G} & m y_{G} & I_{x x} & 0 & 0 \\
m z_{G} & 0 & -m x_{G} & 0 & I_{y y} & 0 \\
-m y_{G} & m x_{G} & 0 & 0 & 0 & I_{z z}
\end{array}\right]^{-1} \cdots
$$

$$
\left[\begin{array}{c}
m v r-m w q+m x_{G}\left(q^{2}+r^{2}\right)-m y_{G} p q-m z_{G} p r \\
m w p-m u r-m x_{G} q p+m y_{G}\left(p^{2}+r^{2}\right)-m z_{G} q r \\
m u q-m v p-m x_{G} r p-m y_{G} r q+m z_{G}\left(q^{2}+p^{2}\right) \\
\left(I_{y y}-I_{z z}\right) q r-m\left[y_{G}(u q+v p)-z_{G}(-w p+u r)\right] \\
\left(I_{x x}-I_{z z}\right) r p-m\left[z_{G}(\dot{u}-v r+w q)-x_{G}(\dot{w}-u q+v p]\right. \\
\left(I_{y y}-I_{x x}\right) p q-m\left[x_{G}(\dot{v}-w p+u r)-y_{G}(\dot{u}-v r+w q]\right.
\end{array}\right]+\left[\begin{array}{c}
0 \\
0 \\
U_{1} \\
U_{2} \\
U_{3} \\
U_{4}
\end{array}\right]++\left[\begin{array}{c}
m g \sin \theta \\
m g \cos \theta \sin \varphi \\
-m g \cos \theta \cos \varphi \\
-m g y_{G} \cos \varphi \cos \theta+m g z_{G} \cos \varphi \cos \theta \\
m g z_{G} \sin \theta+m g x_{G} \cos \varphi \cos \theta \\
-m g y_{G} \sin \theta-m g x_{G} \cos \theta \sin \varphi
\end{array}\right]
$$

\section{KONTROL LQR}

Persmaan matriks state space digunakan untuk mengontrol sudut roll, pitch, dan, yaw. Persamaan state space dapat ditulis seperti pada persamaan (35), dan (36).

$$
\dot{x}=A x+B u
$$




$$
y=C x+D u
$$

Dimana state vector $\mathrm{x}$ dan input vector $\mathrm{u}$ ditulis dalam persamaan (37), dan (38)

$$
\begin{aligned}
& x=[\varphi \theta \psi p q r]^{T} \\
& u=\left[v_{1} v_{2} v_{3} v_{4}\right]^{T}
\end{aligned}
$$

Persamaan gerak nonlinear quadrotor tanpa beban/payload yang harus dilinearkan disekitar kondisi setimbang hover adalah sebagai berikut:

$$
\begin{aligned}
& \dot{\varphi}=\mathrm{p}+\mathrm{q} \tan (\theta) \sin (\varphi)+\mathrm{rtan}(\theta) \cos (\varphi) \\
& \dot{\theta}=\mathrm{q} \cos (\varphi)-\operatorname{rsin}(\varphi) \\
& \dot{\psi}=\mathrm{qsec}(\theta) \sin (\varphi)+\mathrm{rsec}(\theta) \cos (\varphi) \\
& \dot{p}=\frac{I_{Y Y}-I_{Z Z}}{I_{X X}} q r+\frac{U_{2}}{I_{X X}} \\
& \dot{q}=\frac{I_{Z Z}-I_{X X}}{I_{Y Y}} p r+\frac{U_{3}}{I_{Y Y}} \\
& \dot{r}=\frac{I_{X X}-I_{Y Y}}{I_{Z Z}} p q+\frac{U_{4}}{I_{Z Z}}
\end{aligned}
$$

Dari persamaan nonlinear (39), sebuah set persamaan state linear dengan $\mathrm{n}$ variable state $\mathrm{x}_{1}, . ., \mathrm{x}_{\mathrm{n}}$ dan dengan $\mathrm{r}$ variable input $\mathrm{r}_{1}, \ldots, \mathrm{r}_{\mathrm{n}}$, dapat dihitung menggunakan matriks Jacobian. Matriks Jacobian dari sebuah persamaan state dapat dihitung menggunakan persamaan (40) dan (41).

$$
\begin{aligned}
A & =\left[\begin{array}{llll}
\frac{\partial f_{1}}{\partial x_{1}} & \frac{\partial f_{1}}{\partial x_{2}} & \ldots & \frac{\partial f_{1}}{\partial x_{n}} \\
\frac{\partial f_{2}}{\partial x_{1}} & \frac{\partial f_{1}}{\partial x_{2}} & \ldots & \frac{\partial f_{2}}{\partial x_{n}} \\
\ldots & \ldots & \ldots & \ldots \\
\frac{\partial f_{n}}{\partial x_{1}} & \frac{\partial f_{n}}{\partial x_{2}} & \ldots & \frac{\partial f_{n}}{\partial x_{n}}
\end{array}\right]=\left.\frac{\partial f}{\partial x}\right|_{x_{0}, u_{0}} \\
B & =\left[\begin{array}{llll}
\frac{\partial f_{1}}{\partial u_{1}} & \frac{\partial f_{1}}{\partial u_{2}} & \ldots & \frac{\partial f_{1}}{\partial u_{r}} \\
\frac{\partial f_{2}}{\partial u_{1}} & \frac{\partial f_{2}}{\partial u_{2}} & \ldots & \frac{\partial f_{2}}{\partial u_{r}} \\
\ldots & \ldots & \ldots & \ldots \ldots \\
\frac{\partial f_{n}}{\partial u_{1}} & \frac{\partial f_{n}}{\partial u_{2}} & \ldots & \frac{\partial f_{n}}{\partial u_{r}}
\end{array}\right]=\left.\frac{\partial f}{\partial u}\right|_{x_{0}, u_{0}}
\end{aligned}
$$

Persamaan linear state space dari persamaan nonlinear (39) dapat ditulis seperti pada persamaan (42)

$$
\left[\begin{array}{c}
\dot{\varphi} \\
\dot{\theta} \\
\dot{\psi} \\
\dot{p} \\
\dot{q} \\
\dot{r}
\end{array}\right]=\left[\begin{array}{llllll}
A_{11} & A_{12} & A_{13} & A_{14} & A_{15} & A_{16} \\
A_{21} & A_{22} & A_{23} & A_{24} & A_{25} & A_{26} \\
A_{31} & A_{32} & A_{33} & A_{34} & A_{35} & A_{36} \\
A_{41} & A_{42} & A_{43} & A_{44} & A_{45} & A_{46} \\
A_{51} & A_{52} & A_{53} & A_{54} & A_{55} & A_{56} \\
A_{61} & A_{62} & A_{63} & A_{64} & A_{65} & A_{66}
\end{array}\right]\left[\begin{array}{c}
\varphi \\
\theta \\
\psi \\
p \\
q \\
r
\end{array}\right]+\left[\begin{array}{llll}
B_{11} & B_{12} & B_{13} & B_{14} \\
B_{21} & B_{22} & B_{23} & B_{24} \\
B_{31} & B_{32} & B_{33} & B_{34} \\
B_{41} & B_{42} & B_{43} & B_{44} \\
B_{51} & B_{52} & B_{53} & B_{54} \\
B_{61} & B_{62} & B_{63} & B_{64}
\end{array}\right]\left[\begin{array}{c}
v_{1} \\
v_{2} \\
v_{3} \\
v_{4}
\end{array}\right]
$$

Untuk meminimalkan eror yang dihasilkan, state error akan ditambahkan untuk eror sudut roll, pitch, dan yaw. Persamaan output, refernsi, dan eror dapat ditulis dalam persamaan (43), (44), dan (45) 


$$
\begin{gathered}
y=[\varphi \theta \psi]^{T} \\
R=\left[R_{\varphi} R_{\theta} R_{\psi}\right]^{T} \\
e=R-y
\end{gathered}
$$

Persamaan state yang baru dapat diberikan sebagai berikut:

$$
\dot{x}_{1}=e=R-C x
$$

Vektor state yang ditambahkan, persamaan state space dan matriks $\mathrm{C}$ akan menjadi

$$
\begin{gathered}
\bar{x}=\left[\begin{array}{c}
x \\
x_{1}
\end{array}\right] \\
\dot{\bar{x}}=\left[\begin{array}{c}
\dot{x} \\
\dot{x}_{1}
\end{array}\right]=\left[\begin{array}{cc}
A & 0 \\
-C & 0
\end{array}\right]\left[\begin{array}{c}
x \\
x_{1}
\end{array}\right]+\left[\begin{array}{c}
B \\
0
\end{array}\right] u+\left[\begin{array}{l}
0 \\
I
\end{array}\right] R \\
C=\left[\begin{array}{cccccc}
1 & 0 & 0 & 0 & 0 & 0 \\
0 & 1 & 0 & 0 & 0 & 0 \\
0 & 0 & 1 & 0 & 0 & 0
\end{array}\right]
\end{gathered}
$$

Kontrol input dapat diformulasikan dalam persamaan (50)

$$
u=-\bar{K} \bar{x}=\left[K_{1} K_{2}\right]\left[\begin{array}{c}
x \\
x_{1}
\end{array}\right]
$$

Untuk kontrol LQR, Cost functionyang harus diminimumkan diberikan dalam persmaan (51)

$$
J_{L Q R}=\int_{0}^{\infty}\left[\bar{x}^{T} Q \bar{x}+u^{T} R_{u u} u\right] d t
$$

$\mathrm{Q}$ adalah matriks positive semi-definite and $\mathrm{R}_{\mathrm{uu}}$ adalah positive definite matrix

$$
Q=\left[\begin{array}{ccccccccc}
290 & 0 & 0 & 0 & 0 & 0 & 0 & 0 & 0 \\
0 & 290 & 0 & 0 & 0 & 0 & 0 & 0 & 0 \\
0 & 0 & 290 & 0 & 0 & 0 & 0 & 0 & 0 \\
0 & 0 & 0 & 5 & 0 & 0 & 0 & 0 & 0 \\
0 & 0 & 0 & 0 & 5 & 0 & 0 & 0 & 0 \\
0 & 0 & 0 & 0 & 0 & 5 & 0 & 0 & 0 \\
0 & 0 & 0 & 0 & 0 & 0 & 1370 & 0 & 0 \\
0 & 0 & 0 & 0 & 0 & 0 & 0 & 1370 & 0 \\
0 & 0 & 0 & 0 & 0 & 0 & 0 & 0 & 1370
\end{array}\right]
$$

Matriks pembobotan Q dan hubungannya dengan state yang berkaitan dapat disimpulkan seperti pada Tabel 1

Tabel 1. Relasi state dan gain matriks Q

\begin{tabular}{|c|c|c|}
\hline Indeks matriks Q & Gain & State yang berkaitan \\
\hline $\mathrm{Q}(1,1)$ & 290 & Sudut roll \\
\hline
\end{tabular}




\begin{tabular}{|c|c|l|}
\hline $\mathrm{Q}(2,2)$ & 290 & Sudut pitch \\
\hline $\mathrm{Q}(3,3)$ & 290 & Sudut yaw \\
\hline $\mathrm{Q}(4,4)$ & 5 & Roll rate angle state \\
\hline $\mathrm{Q}(5,5)$ & 5 & Pitch rate angle state \\
\hline $\mathrm{Q}(6,6)$ & 5 & Yaw rate angle state \\
\hline $\mathrm{Q}(7,7)$ & 1370 & Eror integral Sudut roll \\
\hline $\mathrm{Q}(8,8)$ & 1370 & Eror integral sudut pitch \\
\hline $\mathrm{Q}(9,9)$ & 1370 & Eror integral sudut yaw \\
\hline
\end{tabular}

Nilai matriks A dan B yang dihasilkan dengan menggunakan Jacobian matriks adalah sebagai berikut

$$
\mathrm{A}=\left[\begin{array}{llllll}
0 & 0 & 0 & 1 & 0 & 0 \\
0 & 0 & 0 & 0 & 1 & 0 \\
0 & 0 & 0 & 0 & 0 & 1 \\
0 & 0 & 0 & 0 & 0 & 0 \\
0 & 0 & 0 & 0 & 0 & 0 \\
0 & 0 & 0 & 0 & 0 & 0
\end{array}\right] \quad \mathrm{B}=\left[\begin{array}{cccc}
0 & 0 & 0 & 0 \\
0 & 0 & 0 & 0 \\
0 & 0 & 0 & 0 \\
0 & -6.0291 & 0 & 5.7388 \\
-5.6078 & 0 & 5.7668 & 0 \\
0.3771 & -0.7054 & 0.5623 & -0.4461
\end{array}\right]
$$

Gain-gain kontrol $\mathrm{K}_{1}$ dan $\mathrm{K}_{2}$ akan lebih mudah dihitung dengan bantuan software MATLAB.

$$
\begin{aligned}
\mathrm{K}_{1} & =\left[\begin{array}{cccccc}
-0.0910 & -4.1037 & 3.9666 & -0.0448 & -0.9322 & 1.8722 \\
-4.0661 & 0.0774 & -4.8128 & -0.8667 & 0.0378 & -2.2248 \\
-0.0899 & 4.0894 & 3.9719 & -0.0438 & 0.8946 & 1.8392 \\
4.0861 & 0.0800 & -4.8890 & 0.9307 & 0.0394 & -2.3104
\end{array}\right] \\
\mathrm{K}_{2} & =\left[\begin{array}{ccc}
0.0768 & 5.5560 & -3.4966 \\
5.6232 & -0.0659 & 4.3332 \\
0.0765 & -5.6031 & -3.5699 \\
-5.5353 & -0.0673 & 4.3041
\end{array}\right]
\end{aligned}
$$

Tabel 2 berisi tentang nilai eigen, damping, dan frekuensi pada closed loop LQR kontrol. Nilai eigen yang paling lambat atau paling dekat dengan Left Half Plane (LHP) adalah -1,73 yang mempunyai rasio damping 1 dan frekuensi $1,73 \mathrm{rad} / \mathrm{s}$.

Tabel 2. Nilai eigen, damping, dan frekuensi dari closed loop LQR

\begin{tabular}{|l|r|r|}
\hline Eigenvalue & \multicolumn{1}{c|}{ Damping } & \multicolumn{1}{c|}{ Frequency } \\
\hline$-4.16 \mathrm{e}+00+3.46 \mathrm{e}+00 \mathrm{i}$ & $7.69 \mathrm{E}-01$ & $5.42 \mathrm{E}+00$ \\
\hline$-4.16 \mathrm{e}+00-3.46 \mathrm{e}+00 \mathrm{i}$ & $7.69 \mathrm{E}-01$ & $5.42 \mathrm{E}+00$ \\
\hline$-4.08 \mathrm{e}+00+3.43 \mathrm{e}+00 \mathrm{i}$ & $7.65 \mathrm{E}-01$ & $5.33 \mathrm{E}+00$ \\
\hline$-4.08 \mathrm{e}+00-3.43 \mathrm{e}+00 \mathrm{i}$ & $7.65 \mathrm{E}-01$ & $5.33 \mathrm{E}+00$ \\
\hline$-1.30 \mathrm{e}+00+1.75 \mathrm{e}+00 \mathrm{i}$ & $5.96 \mathrm{E}-01$ & $2.18 \mathrm{E}+00$ \\
\hline$-1.30 \mathrm{e}+00-1.75 \mathrm{e}+00 \mathrm{i}$ & $5.96 \mathrm{E}-01$ & $2.18 \mathrm{E}+00$ \\
\hline$-1.73 \mathrm{E}+00$ & $1.00 \mathrm{E}+00$ & $1.73 \mathrm{E}+00$ \\
\hline$-2.24 \mathrm{E}+00$ & $1.00 \mathrm{E}+00$ & $2.24 \mathrm{E}+00$ \\
\hline$-2.24 \mathrm{E}+00$ & $1.00 \mathrm{E}+00$ & $2.24 \mathrm{E}+00$ \\
\hline
\end{tabular}

Selanjutnya gain kontrol LQR $\mathrm{K}_{1}$ dan $\mathrm{K}_{2}$ diterapkan untuk mengontrol sudut Euler roll, pitch, dan yaw. Pada Gambar 3, motor model menggunakan persamaan (25) sampai dengan (29), quadrotor nonlinear model menggunakan persamaan dinamika nonlinear (34) pada saat quadrotor mengangkat payload/beban. PID digunakan untuk mengontrol 
ketinggian quadrotor. Sedangkan Gambar 4 merupakan diagram blok control LQR untuk stabilisasi sudut Euler. Integral action digunakan untuk meminimalkan steady state error pada sudut Euler. Parameter dan nilai dari quaadrotor dapat dilihat dalam plampiran.

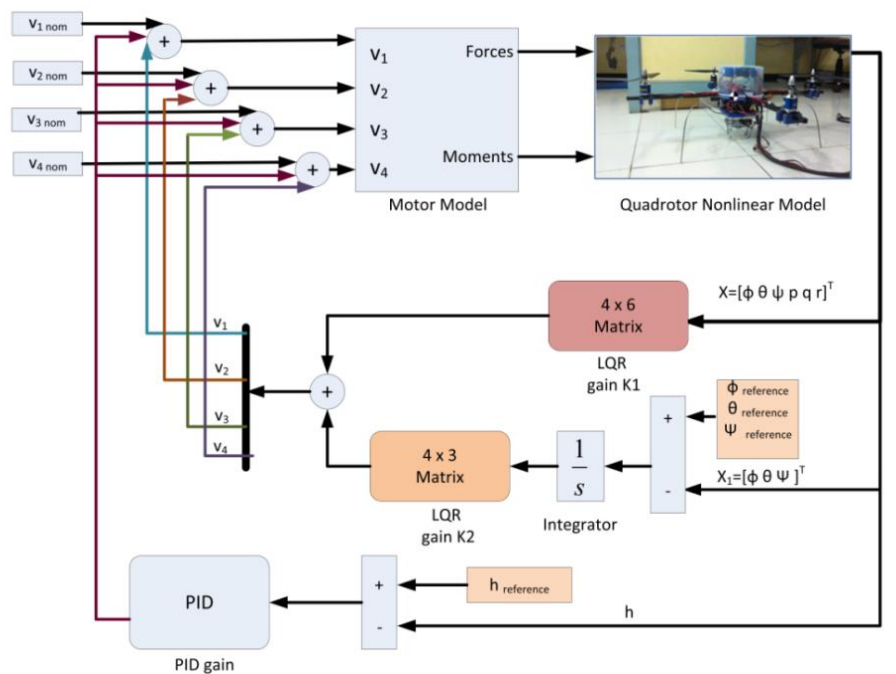

Gambar 3. Diagram blok kontrol LQR

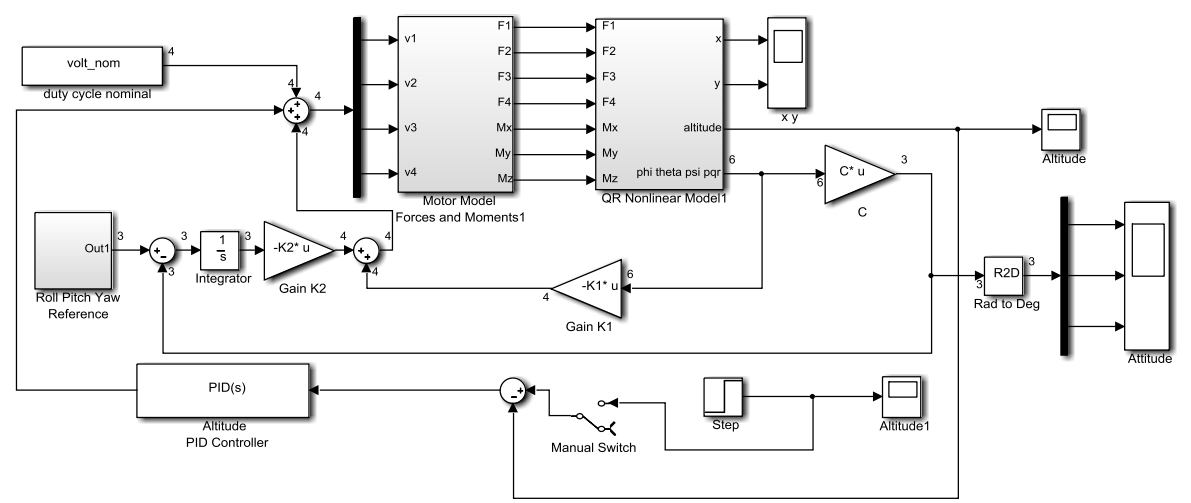

Gambar 4. Diagram blok kontrol LQR untuk stabilisasi sudut Euler dalam Simulink

\section{HASIL DAN KESIMPULAN}

Pada bagian ini akan dilakukan simulasi untuk mengetahui kinerja gain-gain kontrol LQR. Simulasi yang dilakukan dengan memvariasikan massa beban/payload yaitu tanpa beban, beban dengan massa 100 gr, dan beban dengan massa 200 gr. Kemudian variasi beban tersebut akan diterapkan untuk mengontrol sudut Euler quadrotor dengan kondisi sudut awal dan perintah step input. Simulasi dilakukan dengan lama waktu 10 detik. Gambar 5, Gambar, 6, dan Gambar 7 menunjukkan respon sudut roll, pitch, dan yaw dengan kondisi awal $5^{0}$. Respon sudut Euler terhadap kondisi awal menunjukkan bahwa integral action dari kontrol LQR dapat mnghilangkan steady state error. Pada variasi massa beban dari 0 gr sampai dengan 200 gr, steady state error tidak akan muncul.

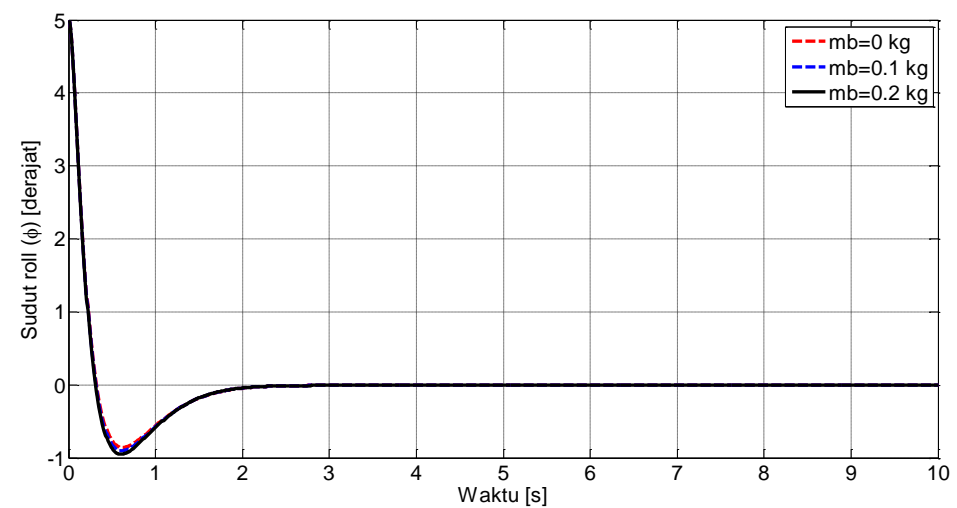

Gambar 5. Respon sudut roll terhadap kondisi awal $5^{0}$ 


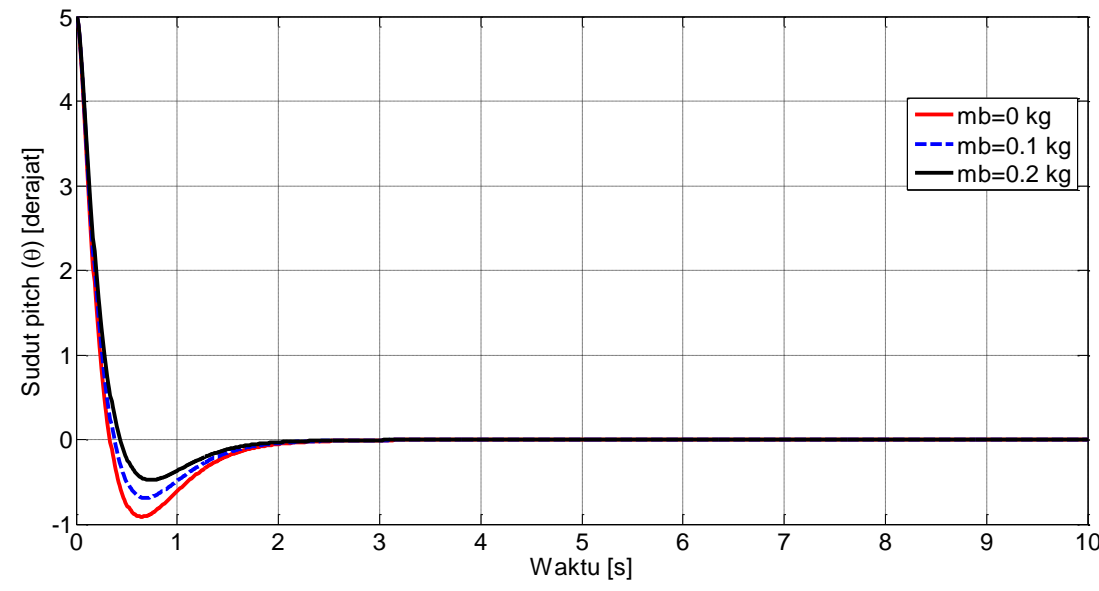

Gambar 6. Respon sudut pitch terhadap kondisi awal $5^{0}$

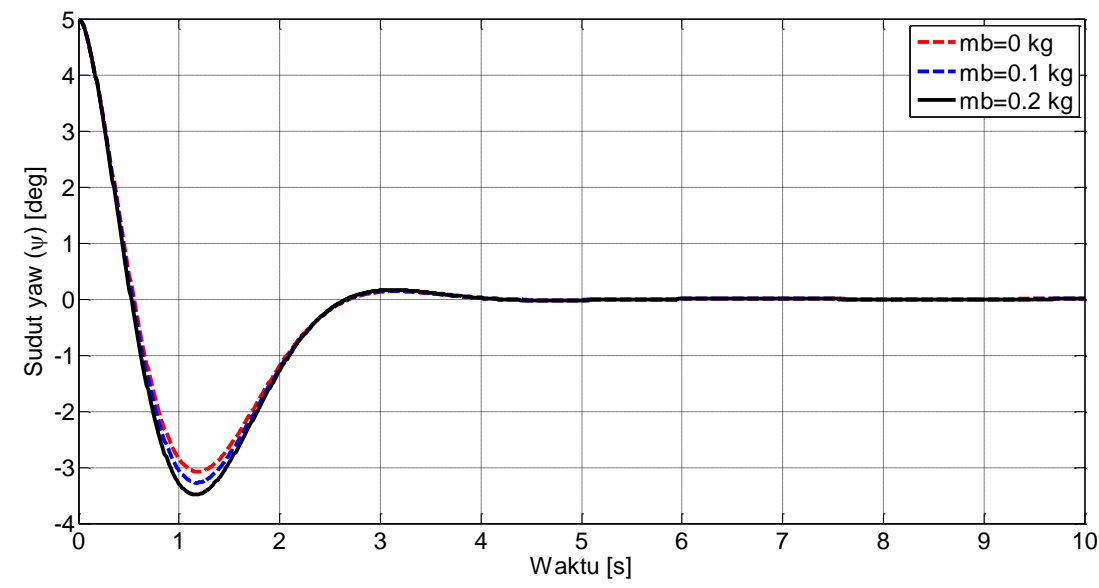

Gambar 7. Respon sudut yaw terhadap kondisi awal $5^{0}$

Respon sudut Euler terhadap perintah step input diberikan pada Gambar 8 dan Gambar 9. Perintah step input dimulai dari 2 detik. Respon sudut yaw lebih lambat dari pada respon sudut roll. Hal ini ditunjukkan dengan besarnya time constant sudut roll yang lebih kecil dari time constant sudut yaw. Respon sudut Euler terhadap step input menunjukkan bahwa integral action dari kontrol LQR dapat mnghilangkan steady state error. Pada variasi massa beban dari $0 \mathrm{gr}$ sampai dengan $200 \mathrm{gr}$, steady state error tidak akan muncul pada perintah step input.

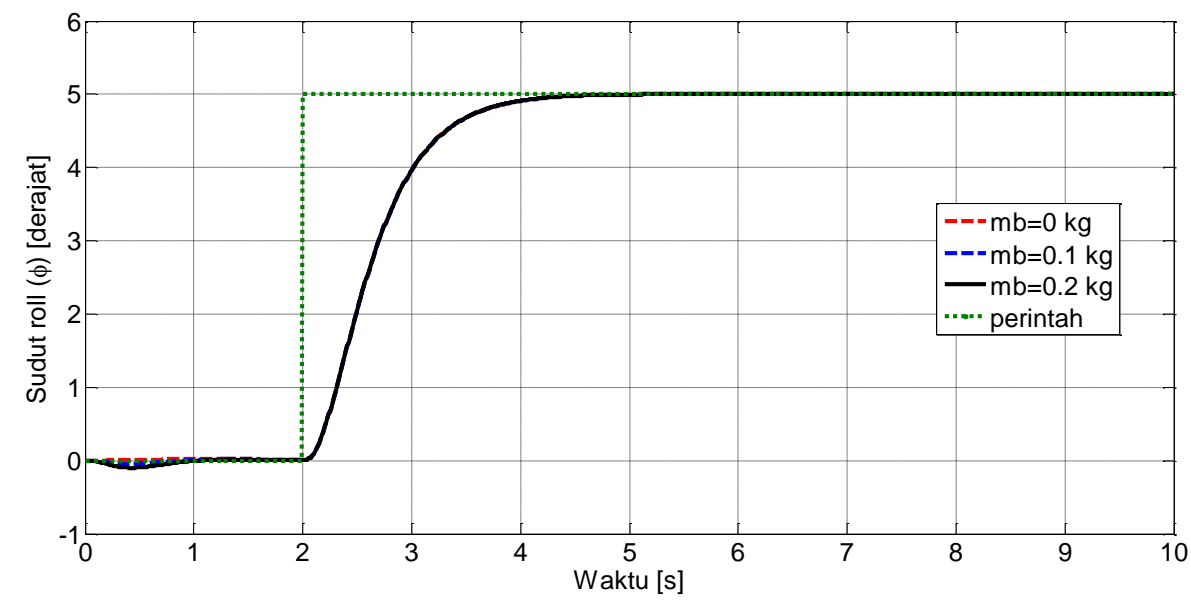

Gambar 8. Respon sudut roll terhadap perintah step input 


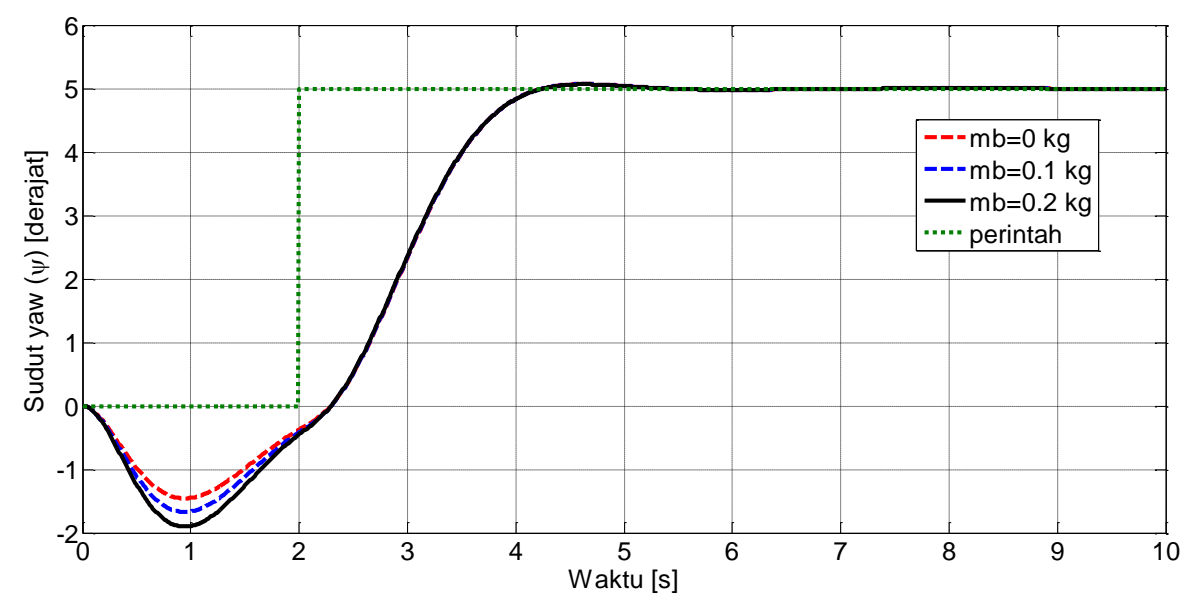

Gambar 9. Respon sudut yaw terhadap step input

Berdasarkan hasil simulasi integral action dari kontrol LQR dapat menghilangkan steady state error setelah diberikan step input, dan kondisi sudut awal sebesar $5^{\circ}$. Variasi beban dari 0 gr sampai dengan 200 gr tidak akan memberikan steady state error.

\section{REFERENSI}

[1] Mellinger, D., Lindsey, Q., Shomin, V, and Vijay K. (2011). Design, Modeling, Estimation and Control for Aerial Grasping and Manipulation. Proceeding of 2011 IEEE/RSJ International Conference on Intelligent Robots and Systems, 2668-2673.

[2] Ghadiok, V. (2011). Autonomous Aerial Manipulation using a Quadrotor. Master Thesis. Utah State University.

[3] Palunko, I., Fierro, R. (2011). Adaptive Control of a Quadrotor with Dynamic Changes in the Center of Gravity. Proceedings of International Federation of Automatic Control (IFAC) World Congress, 18, 2627-2631.

[4] Bouabdallah, S. (2007). Design and Control of Quadrotors with Application to Autonomous Flying. PhD Thesis. Ecole Polytechnique Federal Lausanne.

[5] Bresciani, T. (2008). Modelling, Identification and Control of a Quadrotor Helicopter. Master Thesis. Lund University.

[6] Zao, S. (2004). Advance Control of Autonomous Underwater Vehicles. PhD Thesis. Department of Mechanical Engineering. University of Hawai'i.

[7] LeBas, P. J. (1997). Maximizing AUV Slow Speed Performance. Master Thesis. MIT

[8] Güçlü, A. (2012). Attitude and Altitude Control of an Outdoor Quadrotor. Master Thesis, Atilim University.

[9] Kivrak, A. O. (2006). Design of Control Systems for a Quadrotor Flight Vehicle Equipped with Inertial Sensor. Master Thesis. Atilim University.

\section{UCAPAN TERIMA KASIH}

Penelitian ini didukung oleh Kementrian Riset dan Teknologi, Republik Indonesia, kontrak penelitian Nomor: 38.6/UN7.5/PG/2012 dan Nomor : 39.4/UN7.5/PG/2013 Insentif Riset Sinas.

\section{LAMPIRAN}

Tabel 3. Parameter quadrotor dan nilainya

\begin{tabular}{|c|r|l|}
\hline \multicolumn{2}{|c|}{ Parameter } & \multicolumn{1}{c|}{ Description } \\
\hline$m$ & $1.68 \mathrm{~kg}$ & Quadrotor mass \\
\hline$I_{x x}$ & $0.0264 \mathrm{~kg} \mathrm{~m}^{2}$ & Rolling moment of inertia \\
\hline$I_{y y}$ & $0.0264 \mathrm{~kg} \mathrm{~m}^{2}$ & Pitching moment of inertia \\
\hline$I_{z z}$ & $0.0510 \mathrm{~kg} \mathrm{~m}^{2}$ & Yawing moment of inertia \\
\hline$l$ & $0.285 \mathrm{~m}$ & Horizontal distance: propeller center to CoG of quadrotor \\
\hline$a_{1}$ & $1.5623 \mathrm{~N}$ & Front motor thrust coefficient \\
\hline$a_{2}$ & $1.6797 \mathrm{~N}$ & Right motor thrust coefficient \\
\hline$a_{3}$ & $1.6066 \mathrm{~N}$ & Rear motor thrust coefficient \\
\hline$a_{4}$ & $1.5988 \mathrm{~N}$ & Left motor thrust coefficient \\
\hline
\end{tabular}


Mochammad Ariyanto dkk., Kontrol sudut attitude menggunakan linear quadratic regulator (lqr) untuk quadrotor dengan payload

\begin{tabular}{|l|r|l|}
\hline$b_{1}$ & $-0.2289 \mathrm{~N} / \mathrm{v}$ & Front motor thrust offset \\
\hline$b_{2}$ & $-0.01 \mathrm{~N} / \mathrm{v}$ & Right motor thrust offset \\
\hline$b_{3}$ & $-0.1943 \mathrm{~N} / \mathrm{v}$ & Rear motor thrust offset \\
\hline$b_{4}$ & $-0.3188 \mathrm{~N} / \mathrm{v}$ & Left motor thrust offset \\
\hline$c_{1}$ & $0.0224 \mathrm{~N} . \mathrm{m}$ & Front motor moment coefficient \\
\hline$c_{2}$ & $0.0419 \mathrm{~N} \cdot \mathrm{m}$ & Right motor moment coefficient \\
\hline$c_{3}$ & $0.0334 \mathrm{~N} . \mathrm{m}$ & Rear motor moment coefficient \\
\hline$c_{4}$ & $0.0265 \mathrm{~N} \cdot \mathrm{m}$ & Left motor moment coefficient \\
\hline$d_{1}$ & $-0.0086 \mathrm{~N} \cdot \mathrm{m} / \mathrm{v}$ & Front motor moment offset \\
\hline$d_{2}$ & $-0.0234 \mathrm{~N} \cdot \mathrm{m} / \mathrm{v}$ & Right motor moment offset \\
\hline$d_{3}$ & $-0.0167 \mathrm{~N} \cdot \mathrm{m} / \mathrm{v}$ & Rear motor moment offset \\
\hline$d_{4}$ & $-0.0105 \mathrm{~N} \cdot \mathrm{m} / \mathrm{v}$ & Left motor moment offset \\
\hline
\end{tabular}

